\title{
Covert Orienting of Attention in the Rat and the Role of Striatal Dopamine
}

\author{
Nick M. Ward and Verity J. Brown \\ School of Psychology, University of St. Andrews, St. Andrews KY16 9JU, United Kingdom
}

\begin{abstract}
Attention can be directed to a location in the absence of overt signs of orienting, a phenomenon termed "covert orienting." The ability to orient attention covertly has been well documented in humans, but recent progress has been made with the operational definition of the processes involved in covert orienting. Reaction times to visual targets are quickened when attention is drawn to the location of the subsequent target, and processes such as disengagement, maintenance, and movement of attention can be dissociated by using this method. The possible involvement of striatal dopamine in covert orienting is disputed, with conflicting reports of deficits in covert orienting in patients with Parkinson's disease. To examine the significance of dopamine in the striatum in attentional processes, a
\end{abstract}

test of covert orienting, analogous to that used in humans, was devised for the rat. Unilateral dopamine-depleting lesions of the striatum resulted in increases in mean reaction times contralateral to the side of the lesion, but reaction times did not change differentially as a function of the requirements to maintain, disengage, or shift attention. These findings add additional support to the hypothesis that the deficit that appears as hemineglect observed after striatal damage reflects a motor impairment rather than damage in neural systems underlying mechanisms for directing attention.

Key words: striatum; dopamine; rat; covert orienting; attention; Parkinson's disease
The appearance of a visual target is often associated with the movement of the head and eyes to foveate the attended target; however, shifts of attention can occur in the absence of such overt orienting (Ericksen and Hoffman, 1972; Jonides, 1981). Posner (1980) devised a task to define operationally, in the laboratory, covert orienting of attention: with a subject fixating centrally, a peripheral cue was presented followed by a target light. For $80 \%$ of the trials, the cue corresponded (valid cue) to the side of the subsequent target. For the remaining $20 \%$ of the trials, the cue was presented on the opposite side (invalid cue). Reaction times for trials with a valid cue were faster than for trials with invalid cues. The difference in reaction time between validly cued and invalidly cued trials is referred to as the "validity effect."

Measuring covert shifts in attention, in the absence of overt orienting, has been possible in primates (Petersen et al., 1987; Bowman et al., 1993) but to date has not been reported in other animals, although overt orienting has been demonstrated in pigeons (Shimp and Friedrich, 1993) and the rat (Bushnell and Oshiro, 1994; Bushnell, 1995). The first aim of this study was to provide a description of a paradigm that allows the measurement of covert orienting in the rat.

Posner et al. (1984) proposed that there are three fundamental components to covert orienting, and these have been associated with different brain regions: disengagement of attention (parietal cortex: Posner et al., 1984, 1987; Petersen et al., 1989), shifting of

\footnotetext{
Received Nov. 26, 1995; revised Feb. 13, 1996; accepted Feb. 28, 1996.

This research was supported by The Wellcome Trust (Project Grant 040551/z/94) and the Fujisawa Institute of Neuroscience. N.M.W. is the recipient of a Medical Research Council (UK) Collaborative Studentship (G78/4236) with the Fujisawa Institute of Neuroscience, Department of Pharmacology, University of Edinburgh, Edinburgh FH8 9J7, UK. We thank Dr. T. Phillips for assistance with the behavioral testing, Dr. E. Bowman for helpful discussion, and M. Latimer and the workshop of the School of Psychology for technical support.

Correspondence should be addressed to Dr. Verity J. Brown, School of Psychology, University of St. Andrews, St. Andrews KY16 9JU, UK.

Copyright $\odot 1996$ Society for Neuroscience $0270-6474 / 96 / 163082-07 \$ 05.00 / 0$
}

attention (superior colliculus: Posner and Driver, 1992; Robinson and Kertzman, 1995) and engagement of the new location (lateral pulvinar of the thalamus: Petersen et al., 1987; Rafal and Posner, 1987; Posner and Driver, 1992). Additional experimental and clinical work also has suggested the involvement of anterior cingulate, lateral frontal cortex, and basal ganglia in performance of the task, with these systems thought to contribute to target detection and response preparation (Posner and Driver, 1992).

The dopaminergic antagonist droperidol causes a reduction in the validity effect (Clark et al., 1989), and some studies have reported that Parkinson's disease patients fail to benefit from valid cues (Yamada et al., 1990) or fail to demonstrate a cost after invalid cues (Wright et al., 1990, 1993; Yamada et al., 1990; Bradshaw et al., 1993). In contrast, however, Rafal et al. (1984) and Bennett et al. (1995) found a global increase in reaction time of both valid and invalid trials for Parkinson's disease patients, but with no change in the magnitude of the validity effect. The apparently inconsistent results might be accounted for by heterogeneity of the patient groups: for example, the presence of extra-striate pathology (Javoy-Agid et al., 1981; Fahn, 1986; Agid et al., 1990). The second aim of this study, therefore, was to examine specifically the role of dopamine in the dorsal striatum in covert orienting of attention.

\section{MATERIALS AND METHODS}

Animals. Twenty, pair-housed Lister hooded rats (supplied by in-house breeding program, School of Psychology, University of St. Andrews) were used during the study. The rats were maintained on a $12 \mathrm{hr}$ light/dark cycle with free access to water and a restricted diet of $15-20 \mathrm{gm}$ of sucrose pellets and standard laboratory chow per day (weight range, 215-280 gm at the start and 373-432 gm at completion of the study).

The guidelines laid out in the Principles of Laboratory Animal Care (National Institutes of Health, Publication No. 86-23, revised 1985) and the requirements of the United Kingdom Animals (Scientific Procedures) Act, 1986, were adhered to throughout the study.

Apparatus. The test apparatus was a nine-hole box (Paul Fray, Cam- 
Center Light

\author{
Nose Poke
}

Cue Light

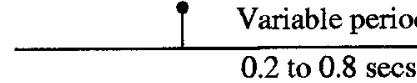

Measures
Valid Cue

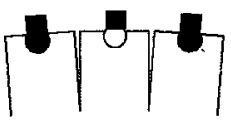

7
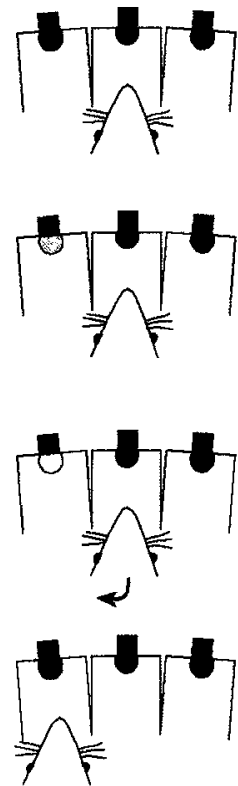

Invalid Cue
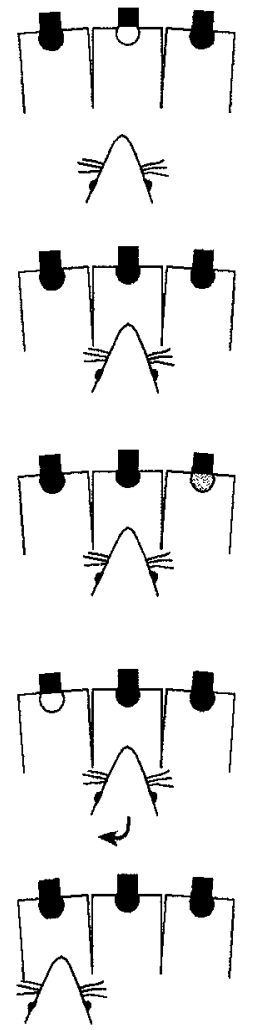

Figure 1. A schematic representation of the covert orienting paradigm in the nine-hole box. At the rear of each hole is a light. In the central hole, the light provides the signal to start a trial. The cue and target are the dim and bright illumination, respectively, of the bulb in the left or right hole. Responses are recorded by nose pokes into the holes, which are monitored by photoelectric cells. Eighty percent of trials consisted of a cue presented on the same side as the subsequent target (valid cue); $20 \%$ of trials consisted of the cue presented on the side opposite the subsequent target (invalid cue).

bridge, UK), which has been described in detail previously (Brown and Robbins, 1989; Carli et al., 1989). The paradigm used only the central array of three holes set in the front wall of the chamber. At the rear of each of the holes was a single bulb that could be switched on and off at different levels of illumination for brief durations under computer control. At the front of each hole there was a photoelectric cell, which registered breaks in an infrared beam at the entrance to the hole. Reaction time was the time from target stimulus onset to the complete withdrawal of the head of the rat from the central hole, measured with an accuracy of $10 \mathrm{msec}$. In adopting this measure, it was accepted that there was a movement time component included in the measure (Carli et al., 1989), with the reaction times extended by the time laken from the start of head withdrawal to the point at which the photoelectric cell beam was no longer interrupted by the head, but this was minimized by the use of nonmechanical switches. In the wall opposite the response holes was a food hopper covered by a hinged panel and containing a light. Pellets (45 mg sucrose; BioServ, Frenchtown, NJ) were delivered to the hopper by a silent operation automatic dispenser and could be heard dropping into the hopper. The chamber was illuminated by a house light in the center of the ceiling. The entire chamber was encased in a sound-attenuating box with a fan that generated low-level background noise and circulated air through the box.

Training regimen. The rats were placed on the restricted diet $24 \mathrm{hr}$ before the commencement of training. Training began with habituation to the test apparatus for $1 \mathrm{hr}$, with standard laboratory food pellets placed in the hopper.

In the first training program, the rat pushed the panel door open to receive a food pellet. During this training, a light in the food hopper was activated with each panel press. Once a rat was able to gain 100 pellets in 15 min (typically, within two 30 min training sessions), it progressed to the next stage of training.

In the next stage of training, the central hole was uncapped. To receive a pellet, the rat now had to place its nose in the central hole, in response to the hole light coming on, and maintain it there for a brief delay, after which the hopper light came on and a food pellet reward was delivered into the food hopper. Premature withdrawal from the central hole resulted in the house light in the chamber switching off for a "time-out" punishment and no food reward; after $1 \mathrm{sec}$, the house light and the light in the food hopper were activated. To initiate a new trial, the rat pushed open the panel door of the food hopper. After $5 \mathrm{~d}$ of this training, the rats were able to wait for delays of $400 \mathrm{msec}$, at which point they progressed to the testing paradigm.

Testing paradigm. Figure 1 illustrates the order of trial events. The three central holes in the chamber were uncapped, and the rat was required to poke its nose into the central hole. After $100 \mathrm{msec}$, the cue was presented. The cue was the brief $(100 \mathrm{msec})$ dim illumination of the bulb in the hole to the left or right of the central hole. After a variable delay of up to $800 \mathrm{msec}$ from the onset of the cue, the target was presented. The target was the bright illumination, for $150 \mathrm{msec}$, of the bulb in one of the holes adjacent to the central hole. To complete the trial, the rat withdrew from the central hole and moved to the location of the target. The cue light indicated the side of the subsequent target on $80 \%$ of the trials (valid cue). The remaining $20 \%$ of trials were invalid cues, in which the cue light appeared on the opposite side of the target. The order of valid and invalid trials and the variable delays between cue and target lights were randomized. The variable delays preceding the target light were increased gradually until they were $200,400,600$, and $800 \mathrm{msec}$. Each testing session lasted until completion of 120 correct trials or for $30 \mathrm{~min}$.

Definition of measures. The time between target light onset and withdrawal from the central hole was recorded as reaction time. The time taken to reach the target hole after withdrawal from the central hole was recorded as movement time.

For the period before and $100 \mathrm{msec}$ after the target light onset, the rat had to keep its nose in the central hole; failure to do so resulted in $1 \mathrm{sec}$ of darkness in the chamber ("time-out") and no food reward and was 
recorded as an early error. Responding with a nose poke in the hole opposite the target light was also punished with a time-out, was unrewarded, and was recorded as an incorrect response. A movement time $>2000 \mathrm{msec}$ also resulted in a time-out and no food reward and was recorded as a late error. In trials that were otherwise correct, reaction times $>1500 \mathrm{msec}$ also were classified as late errors for subsequent analysis, but the trial was rewarded and advanced nevertheless. After a time-out, the trial was repeated until it had been completed successfully.

Collection of behavioral data. Once all the rats had reached the performance criterion of 120 correct trials within $30 \mathrm{~min}$, presurgical data were collected over $5 \mathrm{~d}$ for 10 test sessions $(\sim 1200$ trials). On completion of the collection of presurgical data, the rats were assigned to receive a unilateral intrastriatal infusion of the neurotoxin 6-hydroxydopamine (6-OHDA) (Sigma, Poole, UK). The side that was lesioned was determined by presurgical task performance. If there was an asymmetry in performance, the side contralateral to the strongest validity effect was lesioned $(n=10)$. Where there was no asymmetry, the side of lesion was assigned randomly. After 2 weeks of recovery from surgery, the rats were tested for 3 weeks. A total of $\sim 3900$ correct trials were collected for each rat over this period.

Hypothesis. By using a unilateral model, each rat served as its own control: reaction times for contralateral responses were compared with ipsilateral responses. It is possible to make predictions about the pattern of reaction times expected with different deficits; on the basis of previous neuropsychological studies, many of these are already associated with particular anatomical regions. Table 1 shows the pattern of reaction time change predicted for a unilateral lesion resulting in a given psychological impairment and the anatomical region most likely to be involved. The final two hypotheses are of greatest interest in the current experiment. It is possible that striatal dopamine might play a role in the maintenance of attention (Wright et al., 1990). Alternatively, depletion of striatal dopamine might result in only a response-related deficit.

Surgery. The rats were pretreated with an intraperitoneal injection of the monoamine oxidase inhibitor pargyline $(50 \mathrm{mg} / \mathrm{kg}$ in warm sterile $0.9 \%$ saline; Sigma) before surgery to enhance the efficacy of 6-OHDA (Breese and Traylor, 1971). Twenty minutes after injection, anesthesia was induced in an anesthetic chamber with $4-5 \%$ halothane in an oxygen/nitrous oxide mix (1:3). The rats were then placed in a stereotaxic frame with atraumatic ear bars (Kopf, Tujunga, CA), with the nose bar set at $+5 \mathrm{~mm}$. The halothane was then reduced to $1.5-2 \%$ for the duration of the procedure. A midline incision was made along the scalp, and the skin and fascia were retracted to reveal the skull. A hole was then drilled in the skull at the coordinates $+2.0 \mathrm{~mm}$ anterior and $\pm 3.0 \mathrm{~mm}$ lateral to bregma. A 30 gauge injection cannula containing $8 \mu \mathrm{g}$ of 6-OHDA base in $2 \mu \mathrm{l}$ of ascorbate acid saline was then lowered to $6.5 \mathrm{~mm}$ below skull, and the 6-OHDA was infused manually at a rate of $0.1 \mu \mathrm{l}$ every $10 \mathrm{sec}$. The cannula was left in place for $3 \mathrm{~min}$ before being withdrawn slowly. The incision in the scalp was then closed using sterilized metal clips. Finally, the animal was placed in a warm cage to recover before transfer back to a home cage.

Histology. On completion of postsurgical testing, the rats were killed humanely by intraperitoneal administration of Euthatal $(1.0 \mathrm{ml} / \mathrm{kg}$, pentobarbitone sodium BP $200 \mathrm{mg} / \mathrm{ml}$ ). The rats were perfused transcardially with phosphate buffer for $2 \mathrm{~min}$ at a rate of $10 \mathrm{ml}$ per min, followed by $4 \%$ paraformaldehyde in phosphate buffer for $20 \mathrm{~min}$ at the same rate. The brains then were removed carefully and placed into a $20 \%$ sucrose $/ 4 \%$ paraformaldehyde phosphate buffer solution until processed. Serial coronal sections $50 \mu \mathrm{m}$ thick were cut using a freezing microtome, and two adjacent sections every $400 \mu \mathrm{m}$ were taken for staining with cresyl violet and immunohistochemistry for tyrosine hydroxylase. Tyrosine hydroxylase activity was used as an indirect measure of dopamine depletion in the striatum. To establish the area of tyrosine hydroxylase depletion, the side of lesion was compared with the unlesioned side. Ten sections, at $400 \mu \mathrm{m}$ intervals, were examined between approximately bregma $+1.4 \mathrm{~mm}$ and $-2.4 \mathrm{~mm}$. The area of loss of tyrosine hydroxylase-positive fibers in the head of the caudate-putamen was determined by high-powered microscopy and drawn onto scanned images of the tissue. The scanned images were imported into N.I.H. Image (National Institute of Mental Health, Bethesda, MD), within which analysis of area measurements was performed. The total area of the striatum on these sections was also measured to allow calculation of the percentage of tyrosine hydroxylase depletion in the body of the dorsal striatum (i.e., excluding the ventral striatum and the tail of the caudate). The volume was then calculated by integrating the cross-sectional areas for both depletion and overall striatal area with the distances separating them. Cresyl violet sections were
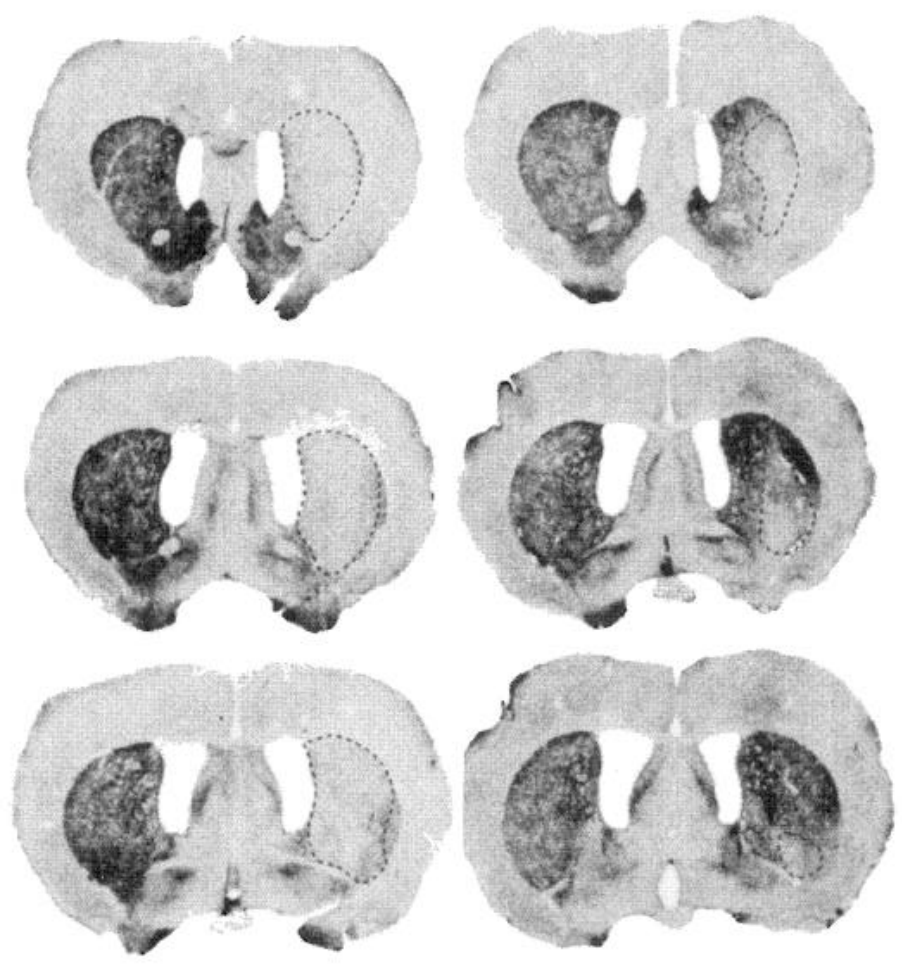

Figure 2. Digitized coronal sections illustrating the extent of tyrosine hydroxylase depletion in the striatum after an intrastriatal injection of the neurotoxin 6-OHDA. The largest lesion is depicted in the sections on the left (rat 289; total area of dopamine depletion in the striatum is $91 \%$ ), and the smallest lesion is depicted in the sections on the right (rat 291; total area of dopamine depletion in the striatum is $19 \%$ ).

also examined under a light microscope for evidence of damage outside the striatum.

Data analysis. Raw data were processed to extract mean reaction times, error-type frequency, and reaction-time distributions for each rat. Accuracy of performance was assessed as the proportion of correct responses as a function of all trials (correct and all error responses). Each type of error also was examined separately as a percentage of all trials.

Mean reaction time, percentage correct, and percentage of errors by type were analyzed by repeated measures ANOVA using the variables surgery, validity, side, and delay. When appropriate, additional investigations of significant interactions were conducted using post hoc Newman-Keuls comparisons.

Probability density distributions (Silverman, 1986; Bowman et al., 1993) of reaction times were produced for the factors side, validity, and surgery. These reaction-time distributions were used to gain insight into the nature of the significant changes in the mean reaction times as indicated by the ANOVA. The distributions were computed by replacing each reaction time with a Gaussian kernel $(\mathrm{SD}=40 \mathrm{msec})$ centered on the reaction time. The kernels were then summed across trials, and the probability of a response per millisecond was plotted against reaction time bins of $10 \mathrm{msec}$.

\section{RESULTS}

\section{Histological results}

Tyrosine hydroxylase depletion was not evident in the striatum of one rat, and therefore this rat was excluded from subsequent analysis. In the remaining rats $(n=19)$, the percentage area of tyrosine hydroxylase depletion in the striatum ranged from $19 \%$ to $91 \%$ (mean 54\%). Figure 2 shows tissue sections stained for tyrosine hydroxylase from the cases with the largest and smallest lesions. The depletion was evident in the body of the striatum between bregma +1.4 and -0.6 , and in the larger lesions it extended into the ventral striatum. The smallest lesion was located centrally in the striatum. Inspection of the cresyl violet 

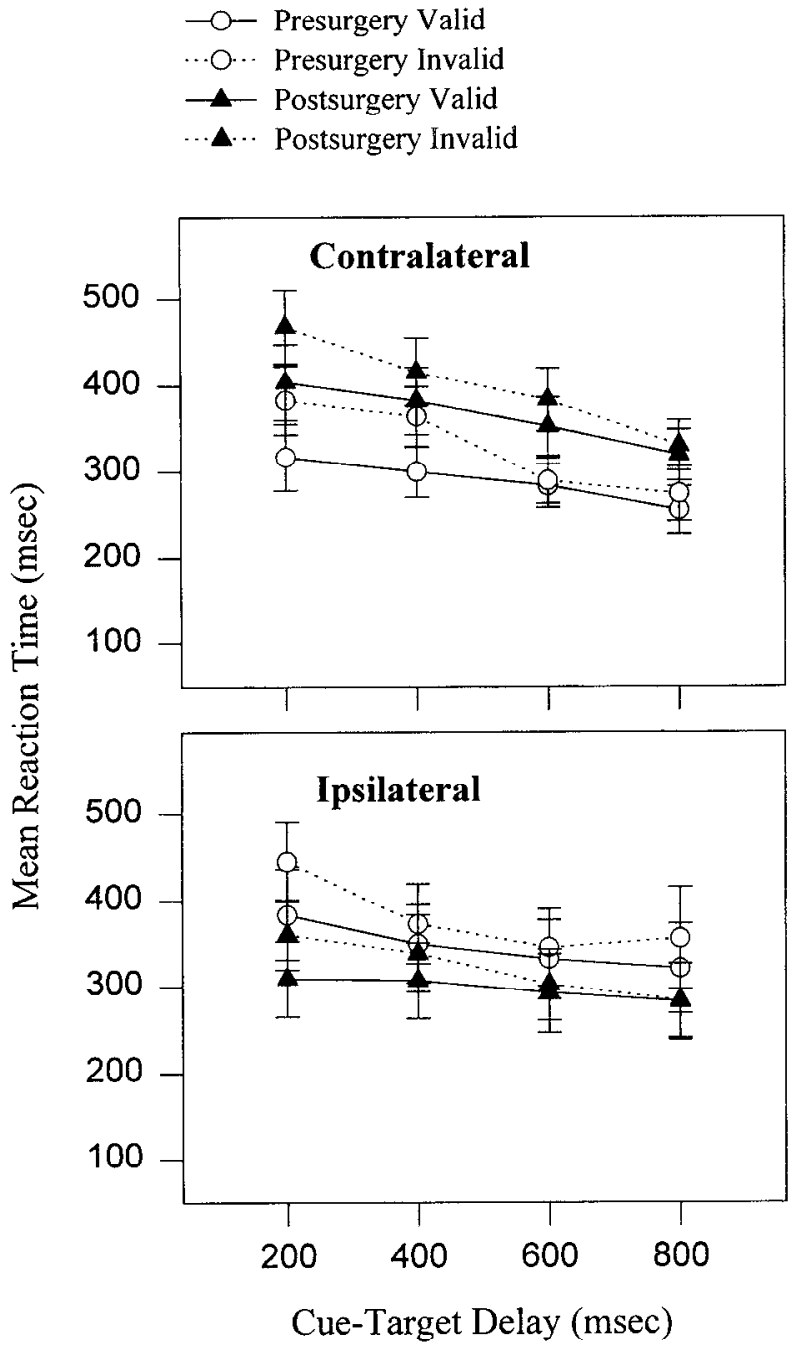

Figure 3. Mean \pm SEM $(n=19)$ of reaction times before and after surgery for validly and invalidly cued trials to each side. Presurgical reaction times are plotted with respect to the side of the subsequent lesion. Mean reaction time was lengthened for contralateral responses postsurgery. Reaction times were longer after invalid as compared with valid cues, and this pattern did not change after unilateral dopamine depletion in the striatum.

sections did not reveal any evidence of damage outside the striatum except for some limited cortical damage attributable to the cannula tract.

\section{Reaction-time performance}

There was a significant validity effect at the shortest two delays (200 and $400 \mathrm{msec}$ ) of 61 and $38 \mathrm{msec}$, regardless of side of response (validity by delay: $F_{(3,54)}=10.63, p<0.0001$ ). The validity effect was not significant at the longer delays of 600 and $800 \mathrm{msec}$.

Figure 3 illustrates the effects of the unilateral striatal dopamine depletion on reaction time. There was no change in the magnitude of the validity effect after surgery (surgery by side by validity: $F_{(1,18)}=0.64$, NS; surgery by validity: $\left.F_{(1,18)}=1.6, \mathrm{NS}\right)$. The mean reaction time, however, increased by an average of 73 msec for all responses initiated contralateral to the side of lesion. Ipsilateral reaction times, by contrast, were faster postoperatively, with a decrease in the mean of $54 \mathrm{msec}$ (surgery by side: $F_{(1,18)}=$ $12.45, p<0.01$ )

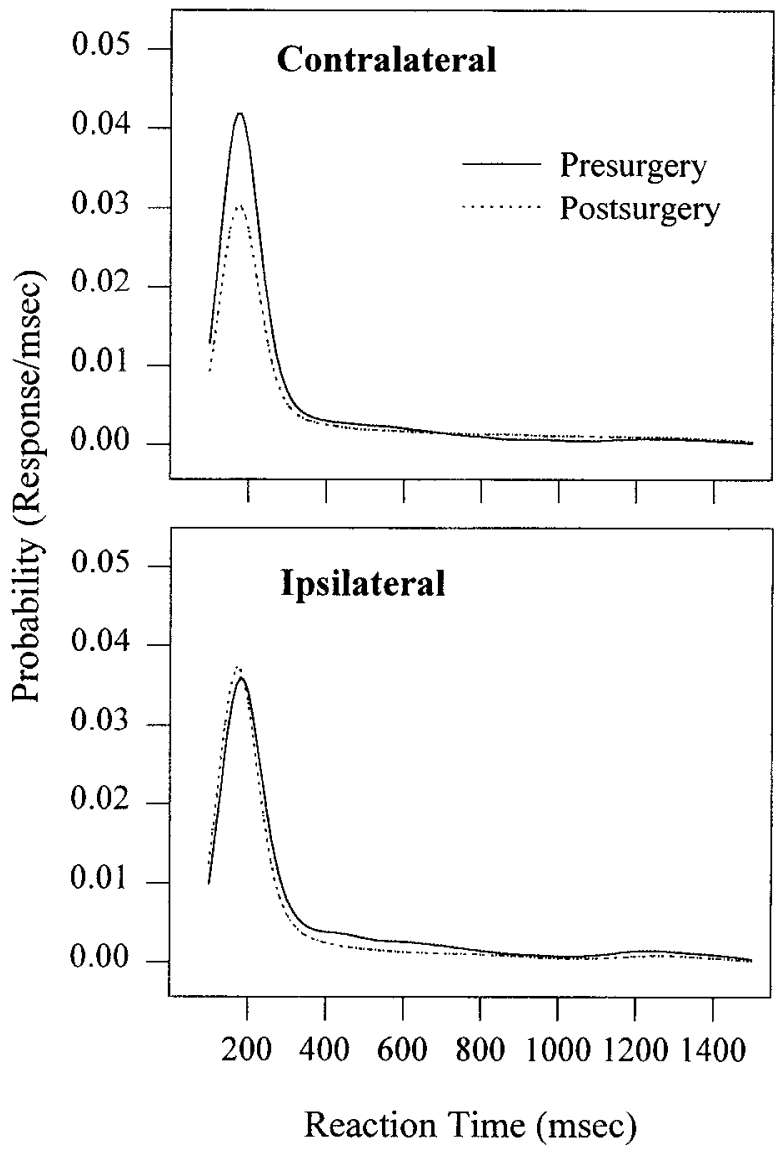

Figure 4. The mean probability density distributions pre- and postsurgery are plotted as a function of response side and include both validity conditions and all cue-target delays. The increase in mean reaction time for responses made contralateral to the side of lesion was attributable to a decrease in the probability of responses occurring at the mode rather than a lateral shift in the distribution.

To test whether there was a relationship between size of the contralateral reaction-time deficit and lesion size, a one-way ANOVA was performed, dividing the group according to lesion size. The reaction-time deficit was greater in the rats with lesion volumes $>50 \%(n=9)$ compared with those with lesion volumes $<50 \%\left(F_{(1,17)}=6.1, p<0.05\right)$.

Figure 4 shows the probability of a response as a function of reaction time. Although there was an increase in mean contralateral reaction time, the average modal reaction time did not change after surgery, remaining at $175 \mathrm{msec}$. The reaction-time distribution for contralateral responses postsurgery, however, displays a downward shift in the probability of a response at the mode. It is apparent that the reason for the significant postsurgery increase in mean reaction time is that the relative frequency of reaction times around the mode has decreased, resulting in the slower reaction times in the tail of distribution increasing the mean. The reaction-time distributions for responses to the ipsilateral side display smaller changes, with both a decrease in the mode $(-10 \mathrm{msec})$ and an increase in the probability of a modal response.

The effect of cue validity at delays of 200 and $400 \mathrm{msec}$ (illustrated in Fig. 5) also causes a small downward shift in the probability of a modal reaction time for invalidly as compared with validly cued trials. This pattern is true for both sides and also for 
Presurgery

Contralateral

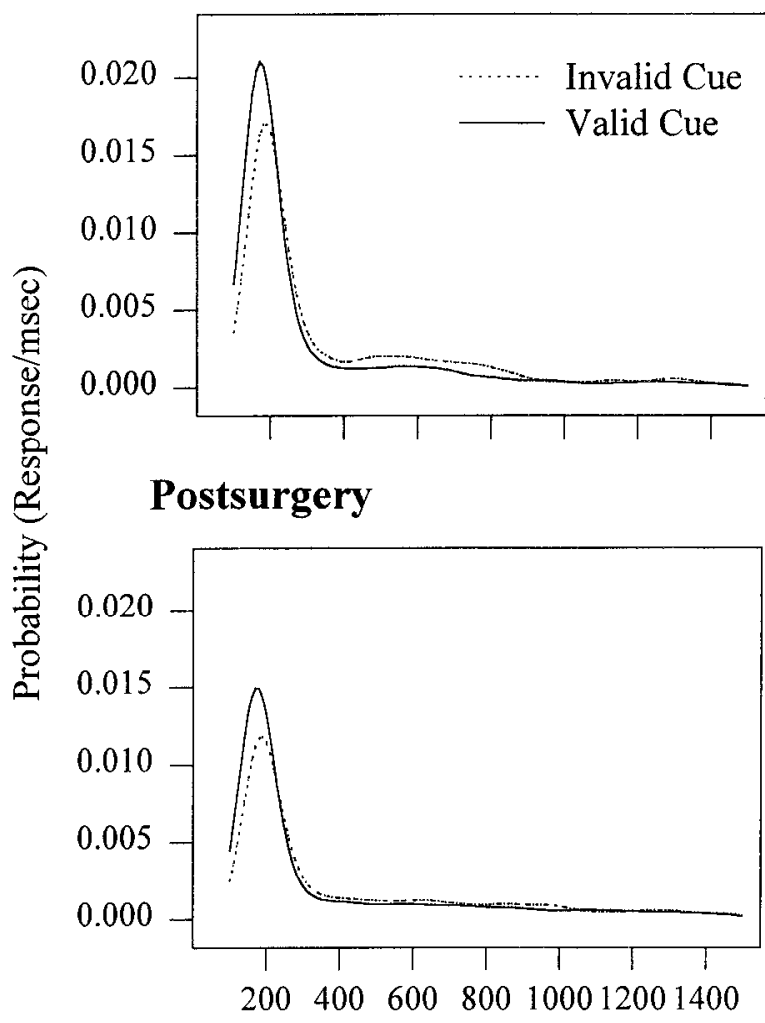

\section{Ipsilateral}
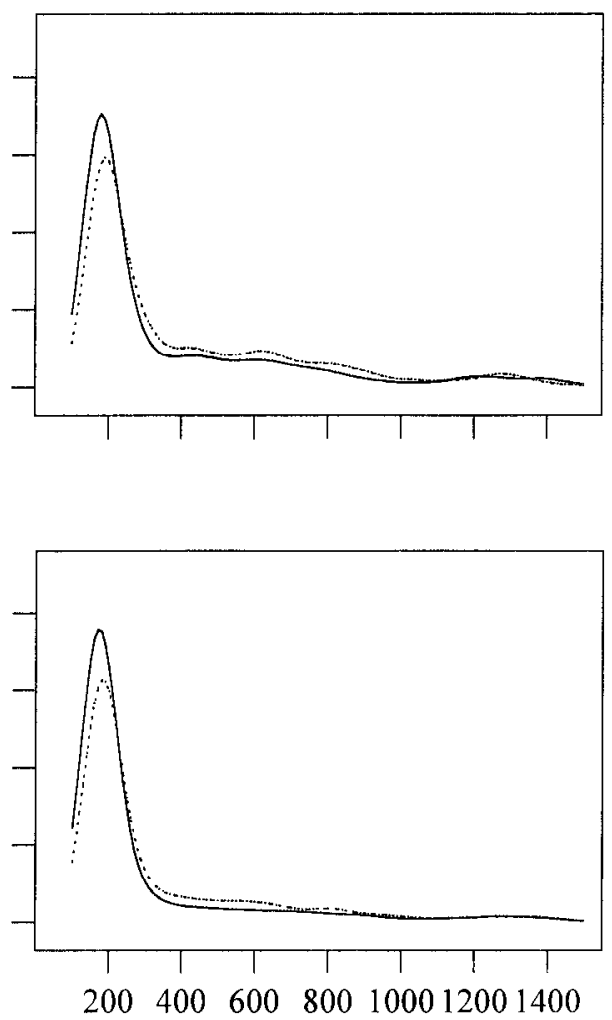

Reaction Time (msec)

Figure 5. The mean probability distributions for the first two delays, plotted as a function of surgery, response side, and validity. The validity effect results in a change in probability of a response at the mode and also a shift in the distribution. This pattern is independent and additive with the effect of surgery.

both presurgery and postsurgery. In addition, there is a lateral shift in the distribution with the mode increasing from $170 \mathrm{msec}$ for valid trials to $190 \mathrm{msec}$ for the invalid trials. Thus, the significant increase in mean reaction times for invalidly cued trials is attributable to a decreased probability of occurrence of responses at the mode and a slight lengthening of reaction times globally.

\section{Percentage correct}

The percentage correct fell from $72 \%$ presurgery to $57 \%$ postsurgery for responses to targets contralateral to the side of lesion. This was greater than the fall in percentage correct postsurgery for responses ipsilateral to the side of lesion (from $69 \%$ to $62 \%$; surgery by side: $\left.F_{(1,18)}=6.53, p<0.02\right)$. The fall in percentage correct was independent of the validity of the cue, with no significant interactions of surgery with validity (surgery by side by validity: $F_{(1,18)}=0.66$, NS; surgery by validity: $F_{(1,18)}=0.34$, NS). The percentage correct also fell as a function of increasing delay $\left(F_{(3,54)}=213.96, p<0.0001\right)$, attributable to an increase in early errors as a function of delay.

The significant interaction between surgery and side for percentage correct was investigated further by examining the percentage of early, incorrect, and late errors. Not surprisingly, there were no interactions of surgery with validity and/or side for early errors (surgery by side by validity $\left(F_{(1,18)}=0.06\right.$, NS; surgery by side: $F_{(1,18)}=0.36, \mathrm{NS}$; surgery by validity: $\left.F_{(1,18)}=0.0003, \mathrm{NS}\right)$;

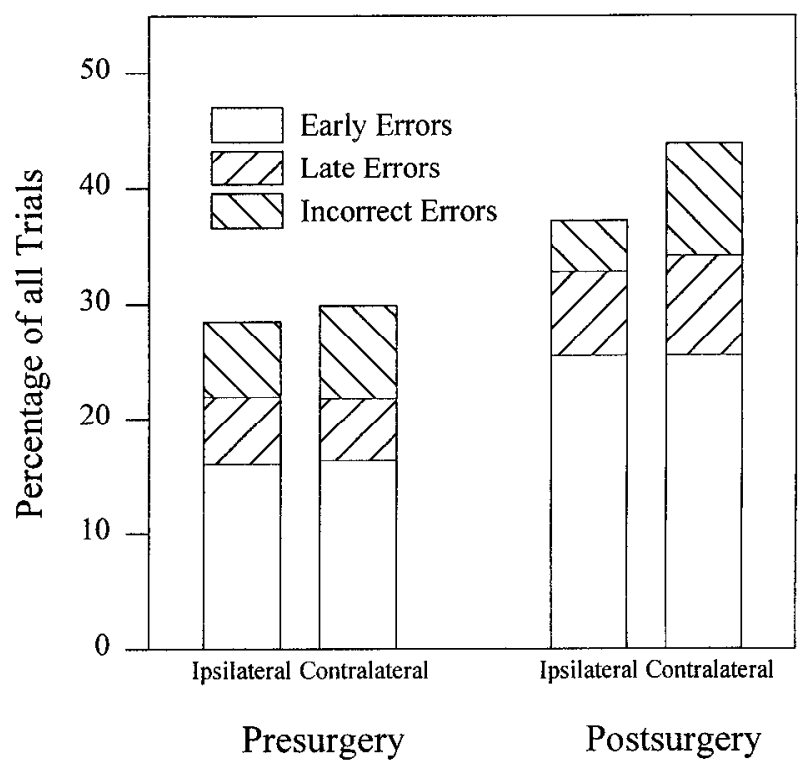

Figure 6. Bar graph showing error type as a percentage of all trials. The significant interaction for percentage correct between side and surgery can be attributed to the cumulative effect of an increase in both late and incorrect errors for responses to the contralateral side. 


\begin{tabular}{|c|c|c|c|c|c|}
\hline Deficit & Anatomical region & $\begin{array}{l}\text { Contralateral } \\
\text { valid }\end{array}$ & $\begin{array}{l}\text { Contralateral } \\
\text { invalid }\end{array}$ & $\begin{array}{l}\text { Ipsilateral } \\
\text { valid }\end{array}$ & $\begin{array}{l}\text { Ipsilateral } \\
\text { invalid }\end{array}$ \\
\hline Disengagement of attention & Posterior parietal cortex ${ }^{a, b}$ & - & $\uparrow$ & - & - \\
\hline Movement of attention & Superior colliculus ${ }^{c, d, c}$ & - & $\uparrow$ & - & $\uparrow$ \\
\hline Engagement of attention & Lateral pulvinar of the thalamus ${ }^{f g}$ & $\uparrow$ & $\uparrow$ & - & $\downarrow$ \\
\hline Maintenance of attention & Striatal dopamine ${ }^{h}$ & $\uparrow$ & - & - & $\downarrow$ \\
\hline Response deficit & Striatum ${ }^{\ell, j, k}$ & $\uparrow$ & $\uparrow$ & - & - \\
\hline
\end{tabular}

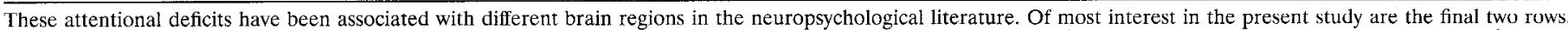

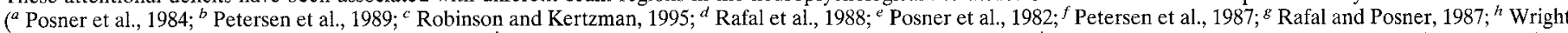

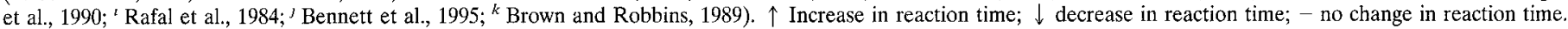

however, the interaction of surgery and side was also not significant for late (surgery by side: $F_{(1,18)}=1.8, \mathrm{NS}$ ) or incorrect $\left(F_{(1,18)}=1.59, \mathrm{NS}\right)$ errors. Nevertheless, as is apparent in Figure 6 , the origin of the significant surgery by side interaction for overall percentage correct is a cumulative effect of an increase in the percentage of both late and incorrect errors postsurgery for responses to the side contralateral to the lesion.

\section{DISCUSSION}

In this study, we have shown that it is possible to measure covert orienting in the rat, thus extending previous demonstrations of covert orienting of attention in humans (Posner, 1980; Posner et al., 1984) and in nonhuman primates (Bowman et al., 1993) to the rat. There was a significant increase in reaction time of responses to targets preceded by an invalid rather than a valid cue. The use of this paradigm in the rat provides a good model in which the neural basis of covert orienting can be investigated further. Although caution must be exercised in assuming that the rat and primate use the same behavioral processes and neural systems in performing this task, nevertheless the similarity of their behavior in this task suggests that they may.

After unilateral striatal dopamine depletion, there was an increase in mean reaction time of responses made to the side contralateral to the lesion (with a corresponding decrease in ipsilateral reaction time), regardless of the validity of the preceding cue. The magnitude of the validity effect did not change after dopamine depletion, which supports the hypothesis that dopamine in the striatum is important for response processes (Table 1) and does not play a role in mechanisms of directed attention.

These results are consistent with and extend the findings of Carli et al. (1989) and Brown and Robbins (1989). Using similar reaction-time paradigms involving responses either away from or toward lateralized sensory stimuli, they reported the consequences of unilateral dopaminc depletion or excitotoxic lesion of the striatum: a bias to respond to the side ipsilateral to the lesion and, after the dopamine depleting lesion, a lengthening of reaction time for responses to the contralateral side. These effects occurred regardless of the side of sensory stimulus. Carli et al. (1989) suggested that there may be attentional changes after the dopamine-depleting lesion. After the lesion, some rats employed an attentional strategy, attending preferentially to a contralateral stimulus that governed an ipsilateral response. Although this observation could be taken as evidence for the "premotor theory" of attention, with attention deriving from motor preparatory processing (Sheliga et al., 1995), the results from the present study, which specifically manipulated attentional processes, have demonstrated that a dopamine-depleting lesion in the striatum has no adverse effect on the covert orienting of attention. Thus, changes in attention after a dopamine-depleting lesion are more likely to be secondary behavioral compensation for lesion effects and are not primary deficits. The present results provide additional evidence that the deficits of orientation termed "neglect" (Marshall and Teitelbaum, 1974), which are commonly observed after depletion of dopamine in the striatum, are attributable to response deficits (Robbins and Brown, 1990) rather than to disturbances in sensory perception or attentional processes.

The increase in mean reaction time does not cause a lateral shift in the entire reaction-time distribution; rather, there is a decreased probability of responses at the mode of the distribution. This finding, of a decrease in the probability of a response at the mode of the distribution, has been reported previously in Parkinson's disease patients tested with and without L-dopa therapy (Brown et al., 1993). The reaction-time distributions of controls and patients with Parkinson's disease were distinguished on the basis of an increase in the proportion of responses with longer reaction times and a decrease in the probability of a response with the modal reaction time. This would suggest that dopamine is particularly significant for fast reaction times.

The suggestion that there might be a role for striatal dopamine in covert orienting has support from several sources. In normal subjects, Clark et al. (1989) reported that the dopamine antagonist droperidol caused decreases in the cost of an invalid cue; however, the specificity of these systemic drug effects has not been determined. Furthermore, Clark et al. (1989) also demonstrated that blockade of noradrenergic function, using clonidine, resulted in similar effects. In patients with Parkinson's disease, Bradshaw et al. (1993) and Wright et al. (1990,1993) suggest that there is a difficulty in maintaining attention, which is manifested as a reduction in the lengthening of reaction time by an invalid cue. Their conclusions, however, were based on a comparison of reaction times in a so-called "neutral cue" condition with reaction times after an invalid cue. Unfortunately, the use of a neutral cue is problematic (Jonides and Mack, 1984). Although it is not informative about target location, the neutral cue may make additional processing demands and may not elicit neutral behavioral strategies in the subject. It is not clear whether the effects reported in the patients are attributable to a reduction in reaction time in the invalid condition or to an increase in reaction time in the neutral condition. Notwithstanding these difficulties, the deficits of the patients may indeed be attributable to a difficulty in maintaining attention, but one arising from dysfunction outside of the striatum as the disease progresses. Consistent with this suggestion, Yamada et al. (1990) reported attentional deficits in patients with Parkinson's disease, but only in patients in the advanced stages. Our present results show that it is unlikely that there is a specific role of dopamine in the striatum in the covert orienting of attention. 


\section{REFERENCES}

Agid Y, Ruberg M, Raisman R, Hirsch E, Javoy-Agid F (1990) The biochemistry of Parkinson's disease. In: Parkinson's disease (Stern GM, ed), pp 99-125. London: Chapman and Hall Medical.

Bennett KMB, Waterman C, Scarpa M, Castiello U (1995) Covert visuospatial attentional mechanisms in Parkinson's discasc. Brain 118:152-166.

Bowman EM, Brown VJ, Kertzman C, Schwarz U, Robinson DL (1993) Covert orienting of attention in Macaques. I. Effects of behavioral context. J Neurophysiol 70:431-443.

Bradshaw JL, Waterfall ML, Phillips JG, Iansek R, Mattingley JB, Bradshaw JA (1993) Re-orientation of attention in Parkinson's disease: an extension to vibrotactile modality. Neuropsychologia 31:51-66.

Breese GR, Traylor TD (1971) Depletion of brain noradrenaline and dopamine by 6-hydroxydopamine. Br J Pharmacol 42:88-99.

Brown VJ, Robbins TW (1989) Elementary processes of response selection mediated by distinct regions of the striatum. J Neurosci 9:3760-3765.

Brown VJ, Schwarz U, Bowman EM, Fuhr P, Robinson DL, Hallett M (1993) Dopamine dependent reaction time deficits in patients with Parkinson's disease are task specific. Neuropsychologia 31:459-469.

Bushnell PJ (1995) Overt orienting in the rat: parametric studies of cued detection of visual targets. Behav Neurosci 109:1095-1105.

Bushnell PJ, Oshiro WM (1994) Overt orienting in the rat: validation of methods and the effects of cholinergic drugs on selective attention. Soc Neurosci Abstr 20:577.

Carli M, Jones GH, Robbins TW (1989) Effects of unilateral dorsal and ventral striatal dopamine depletion on visual neglect in the rat: a neural and behavioural analysis. Neuroscience 29:309-327.

Clark CR, Geffen GM, Geffen LB (1989) Catecholamines and the covert orienting of attention in humans. Neuropsychologia 27:131-139.

Eriksen CW, Hoffman JE (1972) Temporal and spatial characteristics of selective encoding from visual displays. Percept Psychophys 12:201-204.

Fahn S (1986) Parkinson's disease and other basal ganglion disorders. In: Diseases of the nervous system: clinical neurobiology, Vol II (Asbury AK, McKhann GM, McDonald WI, eds) pp 1217-1228. London: William Heinemann Medical Books.

Javoy-Agid F, Ploska A, Agid Y (1981) Microtopography of tyrosine hydroxylase, glutamic acid decarboxylase, and choline acetyltransferase in the substantia nigra and ventral tegmental area of control and Parkinsonian brains. J Neurochem 37:1218-1227.

Jonides J (1981) Voluntary versus automatic control over the mind's eye's movement. In: Attention and performance IX (Long J, Baddeley A, eds) pp 187-203. Hillsdale: Erlbaum.

Jonides J, Mack R (1984) On the cost and benefit of cost and benefit Psychol Bull 96:29-44.
Marshall JF, Teitelbaum P (1974) Further analysis of sensory inattention following lateral hypothalamic damage in rats. J Comp Physiol Psych $86: 375-395$

Petersen SE, Robinson DL, Currie JN (1989) Influences of lesions of parietal cortex on visual spatial attention in humans. Exp Brain Res $76: 267-280$.

Petersen SE, Robinson DL, Morris JD (1987) The contribution of the pulvinar to visual spatial attention. Neuropsychologia 25:97-105.

Posner MI (1980) Orienting of attention. Q J Exp Psychol 32:3-25.

Posner MI, Driver J (1992) The neurobiology of selective attention. Curr Opin Neurobiol 2:165-169.

Posner MI, Cohen Y, Rafal RD (1982) Neural systems control of spatial orienting. Philos Trans R Soc Lond [Biol] 298:187-198.

Posner MI, Walker JA, Friedrich FJ, Rafal RD (1984) Effects of parietal injury on covert orienting of attention. J Neurosci 4:1863-1874.

Posner MI, Walker JA, Friedrich FJ, Rafal RD (1987) How do the parietal lobes direct covert attention? Neuropsychologia 25:135-145.

Rafal RD, Posner MI (1987) Deficits in human visual spatial attention following thalamic lesions. Proc Natl Acad Sci USA 84:7349-7353.

Rafal RD, Posner MI, Friedman JH, Inhoff AW, Bernstein E (1988) Orienting of visual attention in progressive supranuclear palsy. Brain 111:267-280.

Rafal RD, Posner MI, Walker JA, Friedrich FJ (1984) Cognition and the basal ganglia. Brain 107:1083-1094.

Robbins TW, Brown VJ (1990) 'I'he role of the striatum in the mental chronometry of action: a theoretical review. Rev Neurosci 2:181-213.

Robinson DL, Kertzman C (1995) Covert orienting of attention in Macaques. III. Contributions of the superior colliculus. J Neurophysiol 74:713-721.

Sheliga BM, Riggio L, Rizzolatti G (1995) Spatial attention and eye movements. Exp Brain Res 105:261-275.

Shimp CP, Friedrich FJ (1993) Behavioral and computational models of spatial attention. J Exp Psychol Anim Behav Process 19:26-37.

Silverman BW (1986) Density estimation for statistics and data analysis. London: Chapman and Hall.

Wright MJ, Burns RJ, Geffen GM, Geffen LB (1990) Covert orientation of visual attention in Parkinson's disease: an impairment in the maintenance of attention. Neuropsychologia 28:151-159.

Wright MJ, Geffen GM, Geffen LB (1993) Event-related potentials associated with covert orientation of visual attention in Parkinson's disease. Neuropsychologia 31:1283-1297.

Yamada T, Izyuuinn M, Schulzer M, Hirayama K (1990) Covert orienting of attention in Parkinson's disease. J Neurol Neurosurg Psychiatry 53:593-596. 